\title{
'n Faktoriseringsmetode gegrond op 'n additiewe eienskap
}

B. de la Rosa*. H. du T. Mouton, A. van der Westhuizen, D. van Deventer en J.P.H. Wessels

Departement Wiskunde, Universiteit van die Oranje-Vrystaat, Posbus 339, Bloemfontein 9300

Onnzung 2 Augustus 1991; aunuar 22 April 1992

\section{UITTREKSEI.}

Die doel van hierdie artikel is om 'n faktoriseringsmetode te ontwikkel deur van 'n additiewe eienskap van die positiewe he elgetalle gebruik te mak. Aan die hand van bekende resultate word beklemtoon dat die saangestelde, onewe positiewe heelgetalle presies daardie onewe getalle is wat as somme van drie of meer opeenvolgende, positiewe heelgetalle voorgestel kan word. Die direk gë̈mpliseerde algoritmebasis word in hierdie artikel verfyn tot op 'n gewenste peil van optimaliteit. Die resultaat is ' $n$ additiefmultiplikatiewe basis vir 'n algoritme wat gedeeltelik op die genoemde additiewe eienskap en gedeeltelik op langdeling (of alternatiewelik die ggd) berus.

\section{ABSTRACT}

\section{A factorisation method based on an additive property}

The purpose of this article is to develop a method of factorisation based on an additive property of the positive integers. Using known results it is emphasised that the composite odd positive integers are precisely those odd numbers that can be represented as sums of three or inore consecutive positive integers. The obviously implied algorithm basis is refined in this paper up to a desired level of optimality. The result is an additive-multiplicative basis for an algorithm that is based partly on the said additive property and partly on long division (or alternatively the gcd).

\section{Inleiding}

Faktoriseringsmetodes vorm ' $n$ vrugbare bron vir teoretiese besinning sowel as praktiese toepassings. Hierdie stelling word bewys in onder andere die uitstekende oorsig deur Dixon.' Die doel hier is die toevoeging van 'n verdere metode tot die bestaande lys, gegrond op 'n additiewe eienskap van die positiewe heelgetalle. ${ }^{2}$

\section{Somme van opeenvolgende positiewe heelgetalle}

Vir die som in 'n voorstelling van die vorm

$$
t=\sum_{r=1}^{n}(a+r-1)
$$

waar $a \geq 1$ en $n \geq 3$ heelgetalle is, word die term $\delta$-partisie van $\mathrm{t}$ gebruik, en word $\delta(t, a, n)$ daarvoor geskryf. Voorbeelde van $\delta$-partisies is $45=\delta(45,5,6)$ en $45=\delta(45$, $14,3)$. Twee bekende resultate ${ }^{2}$ word hier as grondslag vir die verdere aanbieding aangehaal.

STELLING I: Elke saamgestelde, onewe positiewe heelgetal besit 'n $\delta$-partisie.

Dit volg maklik (as bewys vir hierdie stelling) dat vir enige faktorisering $t=k g(3 \leq k \leq g)$ geld dat

$$
t=\delta\left(t, g-1 /{ }_{2}(k-1), k\right)
$$

Vir enige gegewe faktorisering $t=k g(3 \leq k \leq g)$ volg ook direk dat

$$
t=\delta\left(t, k-1 /{ }_{2}(g-1), g\right) \text { as } g<2 k+1
$$

en

$$
t=\delta\left(t, 1 /{ }_{2}(g+1)-k, 2 k\right) \text { as } g \geq 2 k+1 ;
$$

*Outeur aan wie korrespondensie gerig kan word. en in die geval $g<2 k+1$ geld dat

$$
\delta(t, g-1 / 2(k-1), k) \equiv \delta(t, k-1 / 2(g-1), g) \Leftrightarrow k=g .
$$

Die volgende omgekeerde van STELLING I toon dat die pasbespreekte drie vorms van $\delta$-partisies die enigste is wat ' $n$ onewe positiewe heelgetal kan hê.

STELLING 2: Laat $\delta(t, a, n)$ 'n $\delta$-partisie van 'n onewe positiewe heelgetal $t$ wees, dan geld minstens een van die volgende twee uitkomste:

(1) Daar bestaan 'n faktorisering $t=k g(3 \leq k \leq g, g<2 k$ +1 ) en ooreenstemmend hiermee die versameling $\delta$ partisies

$$
\begin{aligned}
& \Delta(1)=\{\delta(t, g-1 / 2(k-1), k), \delta(t, k-1 / 2(g-1), g)\} \\
& \text { en } \delta(t, a, n) \varepsilon \Delta(1)
\end{aligned}
$$

(2) Daar bestaan 'n faktorisering $t=k g(3 \leq k \leq g, g \geq 2 k$ +1 ) en ooreenstemmend hiermee die versameling $\delta$ partisies

$$
\begin{aligned}
& \Delta(2)=\left\{\delta\left(t, g-1 /{ }_{2}(k-1), k\right), \delta(t, 1 / 2(g+1)-k, 2 k)\right\} \\
& \text { en } \delta(t, a, n) \varepsilon \Delta(2) .
\end{aligned}
$$

GEVOLG: 'n Onewe getal is saamgestel as, en slegs as dit 'n $\delta$-partisie besit.

OPMERKINGS: (1)'n Onewe getal $t$ wat $m$ faktoriserings van die vorm $t=\mathrm{kg}, 3 \leq \mathrm{k} \leq \mathrm{g}$ het, het $2 \mathrm{~m} \delta$-partisies, behalwe in die geval waar $t$ 'n vierkant is, in welke geval 
die aantal $\delta$-partisies gegee word deur $2 m-1$. (2) In enige $\delta$-partisie $\delta(t, a, n)$ is $a \leq 1 / 3(t-3)$, want $a+(a+1)+$ $(a+2)=3 a+3 \leq t$.

Die GEVOLG hierbo word nou eksplisiet geformuleer as:

ALGORITMEBASIS $I$ : ' $n$ Onewe getal $t$ is saamgesteld as, en slegs as daar positiewe heelgetalle $a \leq 1 / 3(t-3)$ en $n \geq$ 3 bestaan sodanig dat $t=\sum_{r=1}^{n}(a+r-1)$. (In hierdie situasie is $t=1 / 2 n(2 a+n-1)$, en: as $n$ onewe is, dan is $n$ ' $n$ faktor van $t$; en as $n$ ewe is, dan is $1 / 2 n$ 'n faktor van $t$.)

Die doel is om hierdie algoritmebasis tot op 'n gewenste peil van optimaliteit te verfyn.

\section{Verfyning van die algoritmebasis}

Uit $\$ 2$ volg dat met elke faktorisering $t=k g(3 \leq k \leq g)$ van 'n onewe positiewe heelgetal $t$ presies twee $\delta$-partisies ooreenstem, naamlik

(1) $\delta(t, g-1 / 2(k-1), k)$

en dan nog presies één van

(2) $\delta(t, k-1 / 2(g-1), g),($ as $g<2 k+1)$

en

(3) $\delta(t, 1 / 2(g+1)-\mathrm{k}, 2 \mathrm{k})$, (as $g \geq 2 k+1)$;

behalwe in die geval waar $k=g$, waar daar slegs die $\delta$ partisie $(1) \equiv(2)$ is. Vir die aanvangsterme in hierdie partisies volg dat

(4) $(g-1 / 2(k-1))-(k-1 / 2(g-1))=3 / 2(g-k) \geq 0$

en

(5) $(g-1 / 2(k-1))-(1 / 2(g+1)-k)=1 / 2(g+k)>0$.

Die volgende notasie is vir die aanvangsterme van die $\delta$ partisies in (I) - (3) gebruik; vir (1):a(kg,k):=g$1 / 2(k-1) ; \operatorname{vir}(2): a(k g, g):=k-1 / 2(g-1) ; \operatorname{vir}(3): a(k g, 2 k)$ $:=1 / 2(g+1)-k$. In hierdie notasie geld nou op grond van STELLING 2, en (4) en (5):

STELLING 3: Laat $t=k g(3 \leq k \leq g)$ 'n faktorisering van 'n onewe getal $t$ wees.

(1) As $g<2 k+1$ word die ooreenstemmende $\delta$-partisies van $t$ gegee deur

$\delta(t, a(k g, k), k)$ en $\delta(t, a(k g, g), g)$,

en geld dat $a(k g, k) \geq a(k g, g)$.

(2)As $g \geq 2 k+1$ word die ooreenstemmende $\delta$-partisies van $t$ gegee deur

$\delta(t, a(k g, k), k)$ en $\delta(t, a(k g, 2 k), 2 k)$,

en geld dat $a(k g, k)>a(k g, 2 k)$.
Vervolgens word die faktoriserings van 'n willekeurige gegewe onewe positiewe heelgetal $t,\{t=k g: 3 \leq k \leq g\}$ beskou, en word vir die doel van rekenaarsoektogte die gunstigste grense vir die aanvangsterme van die ooreenstemmende $\delta$-partisies as funksies van die toetsgetal $t$ bepaal. Eerstens is opgelet dat $a(k g, l) \leq 1 / 3(t-3)$ vir $l=$ $k, g, 2 k$. Verder is:

$a(k g, k)=g-1 / 2(k-1) \geq \sqrt{t}-1 / 2(\sqrt{t}-1)=1 /,(\sqrt{t}+1)$

$a(k g, g)=k-1 / 2(g-1) \leq \sqrt{t}-1 / 2(\sqrt{t}-1)=1 / 2(\sqrt{t}+1)$

$a(k g, 2 k)=1 / 2(g+1)-k=1 / 2(1 / k+1)-k \leq 1 / 2(1 / 3+1)-3=$ $1 / 6(t-15)$.

Die twee bogrense (in die laasgenoemde twee gevalle) is eerste suksesse in die gevalle waar die toetsgetal 'n vierkant van 'n priemgetal of van die vorm $3 p$ ( $p$ priem, $p$ 27 ) onderskeidelik is.

Die gestelde grensafskattings saam met STELLING 2 gee nou:

ALGORITMEBASIS II: Die volgende drie voorwaardes op 'n onewe positiewe heelgetal $t$ is ekwivalent:

(1) $t$ is 'n saamgestelde getal.

(2) Daar bestaan 'n $\delta$-partisie $\delta(t, a, n)$ met $1 / 2(\sqrt{t}+1) \leq$ $a \leq 1 / 3(t-3)$.

(3) Daar bestaan

(3.1) 'n $\delta$-partisie $\delta(t, a, n)$ met $a \leq 1 / 2(\sqrt{t}+1)$ of (3.2) 'n $\delta$-partisie $\delta(t, a, n)$ met $a \leq 1 / 6(t-15)$.

Die $\delta$-partisie in (2) is van die vorm $\delta(k g, a(k g, k), k)$, en dié in (3.1) en (3.2) van die vorms $\delta(k g, a(k g, g), g)$ en $\delta(k g, a(k g, 2 k), 2 k)$ onderskeidelik. Voorwaarde $I /(2)$ is duidelik 'n verbetering op die "rou" ALGORITMEBASIS I. En hierdie basis $I I(2)$ word op sy beurt oortref deur die basis $I /(3)-$ in $(3.1)$ weens die kort interval $\mid 1,1 / 2(\sqrt{t}+1)$ teenoor $[1 / 2(\sqrt{t}+1), 1 / 3(t-3)]$, en in (3.2) weens die kleiner bogrens $1 / 2(t-15)$, sowel as STELLING 3(2) vir gevalle waar $a(k g, 2 k)>1 / 2(\sqrt{t}+1)$. Verder word dus op $A L G O$. RITMEBASIS II(3) gekonsentreer.

Vir groot toetsgetalle $t$ wat slegs oor faktoriserings van die vorm $k g$ met $g \geq 2 k+1$ beskik, kan die soektog na aanvangsterme $a(k g, 2 k)$ oor die (heelgetalle in die) eerste komponent van die toetsinterval

$[1,1 / 6(t-15)]=[1,1 / 2(\sqrt{t}+1)] \cup(1 / 2(\sqrt{t}+1), 1 / 6(t-15)]$

faal, en in die tweede komponent steeds relatief lank duur. Om hierdie probleem te akkommodeer, sal die additiewe basis $I(3)$ vervang word met 'n additief-multiplikatiewe basis waardeur alle soektogte dan binne die interval [ 1 , $1 / 2(\sqrt{t}+1)]$ sal plaasvind. Hiertoe is nodig:

STELLING 4: As $t=k g(3 \leq k \leq g)$ 'n faktorisering met $g$ $\geq 2 k+1$ is, dan is $\mathrm{k}<1 / 2 \sqrt{t}$.

Bewys: $a(k g, 2 k)>1 / 2(\sqrt{t}+1)$

$\Leftrightarrow g+1-2 k>\sqrt{t}+1$

$\Leftrightarrow t+k-2 k^{2}>k \sqrt{t}+k$ 
$\Leftrightarrow 2 k^{2}+\sqrt{t} \cdot k-t<0$

$\Leftrightarrow(k+\sqrt{t})(2 \mathrm{k}-\sqrt{t})<0$

$\Leftrightarrow k<1 / 2 \sqrt{t}$.

ALGORITMEBASIS II(3) kan dus vervang word met:

ALGORITMEBASIS III: Die volgende twee voorwaardes op 'n onewe positiewe heelgetal $t$ is ekwivalent:

(1) $t$ is 'n saamgestelde getal.

(2) Daar bestaan 'n $\delta$-partisie $\delta(t, a, n)$ met $a \leq 1 /,(\sqrt{t}+1)$, of daar bestaan 'n onewe heelgetal $k$ met $3 \leq k<1 / \sqrt{t}$ sodanig dat $k$ 'n faktor van $t$ is.

Die kragpunt van die multiplikatiewe gedeelte van die basis III(2) lê (natuurlik) by toetsgetalle met (onder andere) klein priemfaktore, terwyl dié van die additiewe gedeelte lê by toetsgetalle met faktoriserings van (onder andere) die vorm $k g$ met $k$ in die "omgewing" van $1 / 2 g$. Laasgenoemde word bevestig in:

STELLING 5: Laat $t=k g(3 \leq k \leq g)$ 'n faktorisering van 'n onewe positiewe heelgetal $t$ wees, en $\eta$ 'n positiewe heelgetal. Dan is $a \leq \eta$ vir enige $\delta$-partisie $\delta(t, a, n)$ van $t$ as en slegs as $|2 k-g| \leq 2 \eta-1$.
Bewys: As $g<2 k+1$, dan is $a=a(k g, g)=k-1 / 2(g-1)=$ $1 / 2(2 k-g+1)$ en

$|2 k-g|=2 k-g$, sodat

$|2 k-g| \leq 2 \eta-1 \Leftrightarrow 2 k-g \leq 2 \eta-1 \Leftrightarrow \mathrm{a} \leq \eta$.

As $g \geq 2 k+1$, dan is $a=a(k g, 2 k)=1 / 2(g+1)-k=1 /{ }_{2}(g$ $-2 k+1)$ en $|2 k-g|=g-2 k$, sodat

$|2 k-g| \leq 2 \eta-1 \Leftrightarrow g-2 k \leq 2 \eta-1 \Leftrightarrow \mathrm{a} \leq \eta$.

Die gunstigste toetsgetalle vir die additiewe gedeelte van die algoritmebasis is inderdaad dié van die vorm $k(2 k+1)$ self, want hierin is $a(k g, 2 k)=1$. Die ongunstigste toetsgetalle vir die basis as geheel is die priemgetalle: die basis faal met 'n getal $t$ as en slegs as $t$ 'n priemgetal is! En vlak naas die priemgetalle (in hierdie verband) lê die vierkante van priemgetalle, $t=p^{2}$, want hierin is ' $n$ faktor $k<1 / 2 \sqrt{t}$ nie ter sprake nie, en $a\left(p^{2}, g\right)=1 / 2(\sqrt{t}+1)$.

\section{LITERATUURVERWYSINGS}

1. Dixon, J.D. (1984). Factorization and primality tests, Amer. Math. Monthly, 91: 333-352.

2. De la Rosa, B. (1978). Primes, powers and partitions, The Fibonacci Quarterly, 16: 518-522. 